\section{SAT0237 EFFICACY OF BARICITINIB IN PATIENTS WITH RHEUMATOID ARTHRITIS WHO FAILED 2 OR MORE DMARDS}

M. C. Genovese ${ }^{1}$, M. Dougados ${ }^{2}$, S. Schwartzman ${ }^{3}$, D. Schlichting ${ }^{4}$, S. Beattie ${ }^{4}$, L. Xie ${ }^{4}$, A. Cardoso ${ }^{4}$, J. S. Smolen ${ }^{5} .{ }^{1}$ Stanford University, Palo Alto, United States, ${ }^{2}$ Rene Descartes Univ/Cochin Hospital, Paris, France, ${ }^{3}$ Hospital for Special Surgery (HSS), New York, ${ }^{4}$ Eli Lilly and Company, Indianapolis, United States, ${ }^{5}$ Medical University of Vienna, Vienna, Austria

Background: Baricitinib (Bari) is an oral Janus Kinase (JAK)1/JAK2 inhibitor in development for patients with active rheumatoid arthritis (RA). In Phase 3 studies, Bari has demonstrated clinical efficacy and has a favorable safety profile.

Objectives: To evaluate Bari 2- and 4-mg in patients who have failed multiple DMARDs, across several studies.

Methods: Data from the subgroup of patients who had failed $\geq 2$ DMARDs, including approx. one-half of patients in RA-BUILD (csDMARD-IR) and RA-BEAM (MTX-IR) and all patients from RA-BEACON (bDMARD-IR) were assessed, post hoc, for comparison of Bari 2-mg and 4-mg to placebo across time points using the following measures: ACR20, ACR50, ACR70, SDAI, CDAI, DAS28-CRP, $\mathrm{HAQ}-\mathrm{DI}$, and radiographic assessment of structural damage (mTSS), as well as safety. For patients who had failed $\geq 2$ DMARDs from RA-BEAM, a comparison was also made between Bari 4-mg and adalimumab.

Results: In the $\geq 2$ DMARD-IR populations from RA-BEACON, RA-BUILD, and RA-BEAM, $>95 \%$ had failed MTX as one of the DMARDs. Compared to PBO in this population, Bari resulted in statistically significantly greater improvement in efficacy measures at Week 24 , including physical function (Table). In the $\geq 2$ DMARD-IR population from RA-BEAM (all patients received background MTX), Bari 4-mg was associated with greater improvements compared to adalimumab (table 1 and figure 1). Compared to PBO, Bari 4-mg statistically significantly inhibited structural progression at Week 24 in the $\geq 2$ DMARD-IR subsets of RA-BEAM and RA-BUILD. The overall safety profile of Bari 4-mg in the $\geq 2$ DMARD-IR population was consistent with findings from the overall baricitinib-treated population. ${ }^{¥}$ Data are the proportion (\%) of pts who achieved the response at Week 24 (nonresponder imputation), unless otherwise indicated. ${ }^{\$}$ Approximately $56 \%$ of patients in RA-BUILD and $54 \%$ of patients in RA-BEAM failed $\geq 2$ DMARDs. $\Delta=$ least squares mean change from baseline (modified last observation carried forward). "mTSS data are as randomized using last observation carried forward. ${ }^{*} \mathrm{p} \leq 0.05,{ }^{* *} \mathrm{p} \leq 0.01,{ }^{* * *} \mathrm{p} \leq 0.001$ for Bari 4-mg or Bari 2-mg vs. PBO; ${ }^{*} \mathrm{p} \leq 0.05$ Bari 4mg vs. ADA
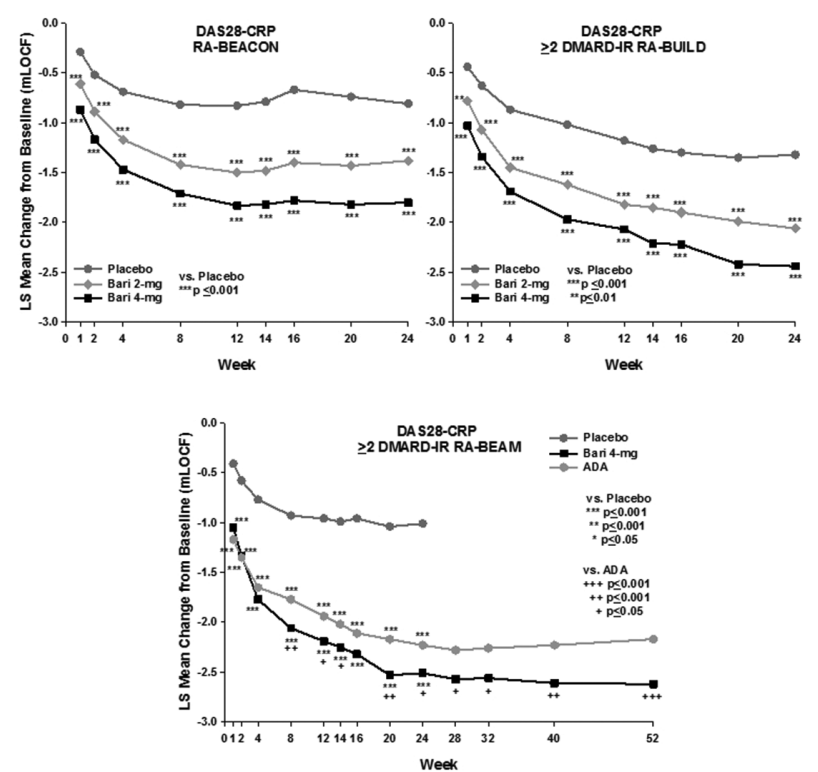

Conclusions: These data demonstrate that a dose response is present between Bari 2-mg and Bari 4-mg, with both doses providing benefit in the patients who failed multiple DMARDs in the phase 3 program by improving signs and symptoms, physical function, and structure.

Disclosure of Interest: M. Genovese Grant/research support from: Eli Lilly \& Company, AbbVie, Consultant for: Eli Lilly \& Company, AbbVie, M. Dougados Grant/research support from: Abbvie, Pfizer, Eli Lilly and Company, Novartis, UCB, Merck, Roche, BMS, Consultant for: Abbvie, Pfizer, Eli Lilly and Company, Novartis, UCB, Merck, Roche, BMS, S. Schwartzman Shareholder of: Amgen, Boston Scientific, Gilead, Medtronic, Consultant for: Abbvie, Crescendo, Dermtech, Genentech, Gilead, Janssen, Eli Lilly and Company, Novartis, Pfizer, Regeneron, Sanofi, UCB, Speakers bureau: Abbvie, Genentech, Janssen, Eli Lilly and Company, Novartis, Pfizer, Regeneron, Sanofi, UCB, Genentech, D. Schlichting Shareholder of: Eli Lilly and Company, Employee of: Eli Lilly and Company, S. Beattie Shareholder of: Eli Lilly and Company, Employee of: Eli Lilly and Company, L. Xie Shareholder of: Eli Lilly and Company, Employee of: Eli Lilly and Company, A. Cardoso Shareholder of: Eli Lilly and Company, Employee of: Eli Lilly and Company, J. Smolen Grant/research support from: AbbVie, Janssen, Eli Lilly and Company, MSD, Pfizer, Roche, Consultant for: AbbVie, Amgen, AstraZeneca, Astro, BMS, Celgene, Celltrion, Chugai, Gilead Glaxo, ILTOO, Janssen, Eli Lilly and Company, Medimmune, MSD, Novartis-Sandoz, Pfizer, Roche, Samsung, Sanofi-Aventis, UCB

DOI: 10.1136/annrheumdis-2018-eular.1889

\begin{tabular}{|l|l}
\hline SAT0238 & RAPID ELEVATION OF ERYTHROCYTE \\
METHOTREXATE-POLYGLUTAMATE3 (MTX-PG3) \\
LEVELS RELATED TO THE EFFICACY OF MTX IN \\
PATIENTS WITH RHEUMATOID ARTHRITIS (RA).
\end{tabular}

M. Nishida ${ }^{1}$, G. Tsuji ${ }^{1}, 2$, M. Takahashi ${ }^{2}$, T. Saitou ${ }^{2}$, Y. Noda $^{1}$, K. Yoneda $^{1}$, N. Amano ${ }^{1}$, S. Sendo ${ }^{1,3}$, A. Onishi ${ }^{3}$, A. Morinobu ${ }^{3}$, M. Shinohara $^{4,5}$, S. Kumagai ${ }^{1}, 2$. ${ }^{1}$ Center for Rheumatic Diseases, Shinko Hospital, ${ }^{2}$ Shinko Institute for Medical Research, ${ }^{3}$ Department of Rheumatology and Clinical Immunology, ${ }^{4}$ Division of Epidemiology, ${ }^{5}$ The Integrated Center for Mass Spectrometry, Kobe University Graduate School of Medicine, Kobe, Japan

Background: MTX is transported into cells and retained long after polyglutamation. Recently, it has been described that the response to MTX treatment is related to the intracellular MTX-polyglutamate (MTX-PG) levels, but little is known about its details and determinants ${ }^{1)}$

Objectives: To clarify the association of erythrocyte concentrations of MTX-PGs (PG1 to PG4) with the efficacy of MTX in 35 MTX naïve patients with RA.

Methods: We measured erythrocyte MTX-PGs at $4,12,24$, and 48 weeks after administration of MTX using liquid chromatography (LC)-mass spectrometry (MS)/MS assay. The associations of MTX-PG concentrations with disease activity and the 9 SNPs including 5 SNPs reported for associations with MTX-efficacy were analyzed.

Results: As shown in the figure, MTX-PG1 and PG2 elevated at week 4, and total MTX-PGs as well as MTX-PG3 and MTX-PG4 increased until week 12. The MTX-PG2 fraction (as percentage of total) was $26 \%$ to $29 \%$ and almost constant throughout the course. MTX-PG3 and MTX-PG4 fractions were gradually elevated over time, although MTX-PG1 fraction was decreased. A negative association of Disease Activity Score in 28 joints (DAS28) at week 4, 12 and 24 was observed with levels of MTX-PG2 ( $p=0.008)$, MTX-PG3 $(p=0.0045)$, MTX-PG4 $(\mathrm{p}=0.0142)$ and total MTX-PG $(\mathrm{p}=0.023)$. On the other hand, $\triangle \mathrm{DAS} 28$ (change in DAS28 scores from baseline) was positively correlated to fraction of MTX-PG3 $(p=0.011)$ but negatively correlated to that of MTX-PG1 $(p=0.0071)$. At week 12 and 24, MTX-PG2 fraction was higher $(p=0.0783)$ in the patients who achieved the EULAR good response criteria than in those who did not.

MTX-PG3 levels were associated with SLC19A1c.80G $>A$ and FMO2 c.585A>G, and the fraction of MTX-PG2 in total MTX-PGs was associated with GGH c. $452 \mathrm{C}>\mathrm{T}$ and with $\mathrm{FMO} 2 \mathrm{c} .585 \mathrm{~A}>\mathrm{G}$.

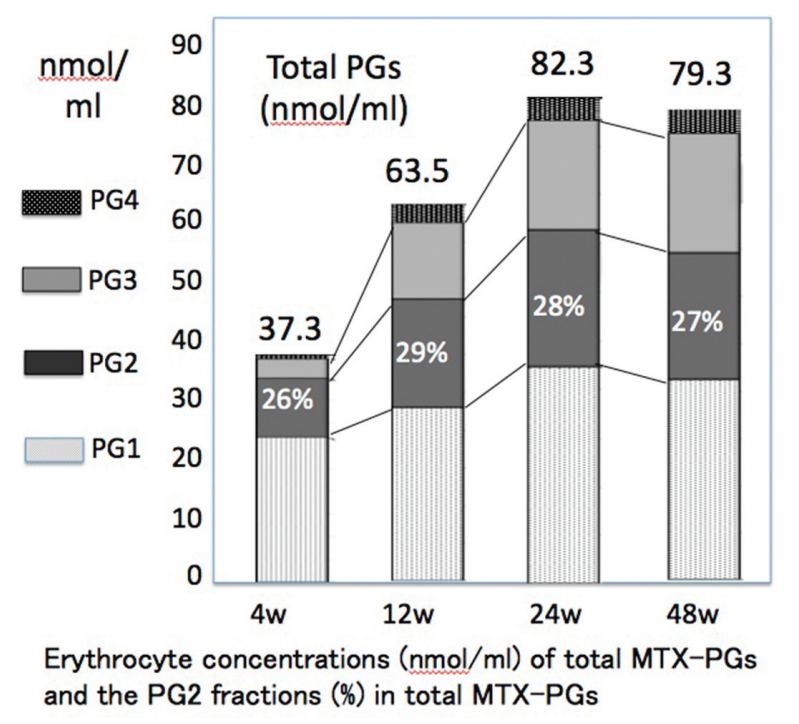

Conclusions: This study suggests that rapid elevation of erythrocyte MTX-PG3 levels from MTX-PG1 through MTX-PG2 and higher distribution of MTX-PG3 is 